\title{
Visceral-to-subcutaneous fat ratio is a possible prognostic factor for type 1 endometrial cancer
}

\author{
Michiko Wada ${ }^{1,2} \cdot$ Ken Yamaguchi $^{1,2,3}$ (D) Hajime Yamakage ${ }^{1} \cdot$ Takayuki Inoue $^{1} \cdot$ Toru Kusakabe $^{1} \cdot$ Kaoru Abiko $^{2}$. \\ Kenji Takakura $^{2} \cdot$ Ikuo Konishi $^{2} \cdot$ Noriko Satoh-Asahara $^{1}$
}

Received: 2 August 2021 / Accepted: 19 October 2021 / Published online: 30 October 2021

(c) The Author(s) 2021

\begin{abstract}
Background Associations have been observed between obesity defined by the body mass index (BMI) and the incidence of endometrial cancer. However, the impact of obesity on the prognosis of endometrial cancer is not yet clear. Recently, visceral fat has been considered to have a greater impact on malignant disease in obese patients than subcutaneous fat. In this study, we investigated the association between prognostic factors of type 1 and type 2 endometrial cancer and obesity parameters. Methods The impacts of clinical factors on the progression-free survival (PFS) and overall survival (OS) were analyzed retrospectively in 145 primary endometrial cancer patients. The factors included age, BMI, pathological findings, Federation of Gynecology and Obstetrics (FIGO) stage, status of lymph node metastasis, and the amounts of visceral and subcutaneous fat obtained from computed tomography (CT) data.

Results Only the visceral-to-subcutaneous fat ratio ( $V / S$ ratio) (cutoff value 0.5 ) corresponded to a significant difference in OS and PFS in type 1 endometrial cancer $(p=0.0080, p=0.0053)$ according to the results of log-rank tests of Kaplan-Meier curves. The COX regression univariate analysis revealed that only the $V / S$ ratio was a significant prognostic factor for PFS, but not $\mathrm{OS}$ ( $p=0.033$ and $p=0.270$, respectively).

Conclusion A $V / S$ ratio $>0.5$ is a possible factor for poor prognosis in type 1 endometrial cancer. Further research is needed to investigate the preventive and therapeutic effects of reducing visceral fat on the prognosis of this type of cancer.
\end{abstract}

Keywords Endometrial cancer $\cdot$ Visceral fat $\cdot$ Subcutaneous fat $\cdot$ Prognosis

\section{Introduction}

Endometrial cancer is the most common gynecologic cancer. Its incidence is increasing over time and in successive generations in several countries, especially in South Africa and several countries in Asia, including Japan, where rapid

Ken Yamaguchi

soulken@kuhp.kyoto-u.ac.jp

1 Department of Endocrinology, Metabolism, and Hypertension, Clinical Research Institute, National Hospital Organization Kyoto Medical Center, 1-1 Mukaihata-cho, Fukakusa, Fushimi-ku, Kyoto 612-8555, Japan

2 Department of Obstetrics and Gynecology, National Hospital Organization Kyoto Medical Center, Kyoto 612-8555, Japan

3 Department of Gynecology and Obstetrics, Kyoto University Graduate School of Medicine, 54 Kawahara-cho, Shogoin, Sakyo-ku, Kyoto 606-8507, Japan socioeconomic transitions are occurring [1,2]. Endometrial cancer is divided into type 1 and type 2 . Type 1 is usually not very aggressive and estrogen dependent, whereas type 2 is more aggressive than type 1 and consists of all other forms of endometrial cancer that do not fall under type 1 . The relation between obesity and the development of endometrial cancer is reported to be caused by unopposed estrogen. Estrogen is generated from testosterone by aromatase in adipose cells, which makes obesity a risk factor for type 1 endometrial cancer [3]. On the other hand, the levels of sex hormones are not different between type 1 and type 2, suggesting that type 2 is related to estrogen [4].

Obesity is associated with the incidence of malignant disease [5-7]. Several parameters are used to evaluate obesity, including the body mass index (BMI), abdominal circumference (AC), and visceral-to-subcutaneous fat ratio ( $V / S$ ratio), among others [2]. In particular, visceral fat is an important risk factor for the morbidity and mortality of several cancers 
[8], and the incidence of endometrial cancer has increased with the occurrence of the obesity epidemic [9].

Visceral obesity measured by ultrasonography is strongly related to the incidence of endometrial cancer [10]. Obesity could possibly influence the prognosis and incidence of endometrial cancer, but information about its impact on the prognosis is limited [11]. Thus, this study investigates the association between prognostic factors of type 1 and type 2 endometrial cancer and obesity parameters, especially the $V / S$ ratio.

\section{Materials and methods}

\section{Study design}

This retrospective study was approved by the ethics committee of National Hospital Organization Kyoto Medical Center. Institutional Review Board number is 17-061. We searched the pathological database system of the medical center to identify patients who had endometrial cancer from January 2012 to December 2016. The eligibility criterion was a diagnosis of primary uterine endometrial cancer that had been confirmed pathologically. The exclusion criteria were (1) recurrent disease, (2) no surgery, chemotherapy, or radiation therapy, and (3) a lack of computed tomography (CT) images at diagnosis. Ultimately, 145 primary endometrial cancer patients satisfied the eligibility criteria and were enrolled.

The clinical factors examined included age, BMI, pathological findings, Federation of Gynecology and Obstetrics (FIGO) stage, status of lymph node metastasis, amounts of visceral and subcutaneous fat obtained from CT data, progression-free survival (PFS), and overall survival (OS). FATSCAN $^{\circledR}$ was used for the analysis of visceral and subcutaneous fat. CT images at the level of the umbilicus were analyzed to measure visceral fat, subcutaneous fat, and abdominal circumference (AC). The $V / S$ ratio was calculated to evaluate visceral fat obesity.

\section{Statistical analyses}

The association between two parameters was evaluated by the Mann-Whitney $U$ test, while the chi-squared test was used for trend analysis of the contingency table of FIGO stages. PFS was calculated as the time in which the patient was free of cancer after a particular treatment, while OS was calculated as the duration from the date of the first treatment until the date of death or the last follow-up. The Kaplan-Meier method was used to analyze PFS and OS. We used a log-rank test and COX-regression univariate analysis to assess the association between clinical factors and prognosis. The statistical analyses were performed in SPSS (Version 25.0, Inc., Chicago, IL, USA), and $p<0.05$ was considered statistically significant.

\section{Results}

\section{Patient characteristics}

Table 1 shows the characteristics of endometrial cancer patients. There were 96 cases of type 1 and 52 cases of type 2 . The age of the type 1 cases was significantly lower than that of type 2 patients (median \pm standard deviation (SD) $55.5 \pm 12.5$ versus $66.0 \pm 9.8$ years old; $p<0.05$ ). Type 2 cases showed a significantly higher rate of positive results for ascites cytology, lymph vascular involvement, lymph node metastasis, recurrence, and death ( $p<0.05$ for all). There were no statistically significant differences between type 1 and type 2 patients in terms of BMI, AC, total fat area, visceral fat area, subcutaneous fat area, V/S ratio, familial history of malignancy complication with diabetes mellitus, and complication with hypertension $(p=0.41,0.65$, $0.33,0.16,0.49,0.28,0.85,0.58$, and 1.00 , respectively). There were no cases that died from these complications.

\section{Prognostic impacts of obesity parameters}

Figure 1 shows a scatter diagram of the obesity parameters. We analyzed the association between BMI and other obesity parameters. There was a strong correlation between BMI and all types of fat $(R=0.87, p<0.01$; Fig. 1a), visceral fat $(R=0.67, p<0.01$; Fig. $1 \mathrm{~b})$, subcutaneous fat $(R=0.87$, $p<0.01$; Fig. 1c), and AC ( $R=0.88, p<0.01$; Fig. $1 \mathrm{~d})$. However, BMI and $V / S$ ratio did not show a significant correlation $(R=0.05, p=0.52$; Fig. $1 \mathrm{e})$.

We performed log-rank tests on OS and PFS for each type of endometrial cancer in terms of BMI (cutoff value $25 \mathrm{~kg} / \mathrm{m}^{2}$; Figs. 2a, b and 3a, b; PFS and OS, respectively), AC (cutoff value $100 \mathrm{~cm}$; Figs. 2c, d and 3c, d; PFS and $\mathrm{OS}$, respectively), and $V / S$ ratio (cutoff value 0.5 ; Fig. $2 \mathrm{e}$, f, 3e, f; PFS and OS, respectively). For PFS, Kaplan-Meier curves of type 1 and type 2 endometrial cancer showed no significant difference between BMI $>25 \mathrm{~kg} / \mathrm{m}^{2}$ and $<25 \mathrm{~kg} /$ $\mathrm{m}^{2}$ ( $p=0.6344$ and $p=0.5359$, respectively; Fig. 2a, b). AC showed no significant difference between $\mathrm{AC} \geq 100 \mathrm{~cm}$ and $<100 \mathrm{~cm}$ in both type 1 and type 2 endometrial cancers ( $p=0.3718$ and $p=0.5791$, respectively; Fig. $2 c, d)$. However, Kaplan-Meier curves of the V/S ratio identified a significant difference between $V / S$ ratios $>0.5$ and $<0.5$ in 
Table 1 Characteristics of endometrial cancer patients

\begin{tabular}{lllll}
\hline & All $(n=148)$ & Type 1 $(n=96)$ & Type 2(n=52) & $\begin{array}{l}p \text { value } \\
\text { type } 1 \\
\text { vs. } 2)\end{array}$ \\
\hline Age of diagnosis (median) & & & & $<0.05^{*}$ \\
BMI $\left(\mathrm{kg} /\left(\mathrm{m}^{2}\right)(\right.$ median$)$ & 61.5 & 55.5 & 66.0 & 0.41 \\
AC $(\mathrm{cm})\left(\mathrm{median}^{2}\right.$ & 23.5 & 23.6 & 23.5 & 0.65 \\
Total fat area $\left(\mathrm{cm}^{2}\right)($ median$)$ & 84.8 & 84.8 & 84.5 & 0.33 \\
Visceral fat area $\left(\mathrm{cm}^{2}\right)($ median) & 250.0 & 255.6 & 220.9 & 0.16 \\
Subcutaneous fat area $\left(\mathrm{cm}^{2}\right)($ median$)$ & 176.62 & 82.1 & 67.2 & 0.49 \\
V/S ratio (median) & 0.38 & 183.6 & 138.3 & 0.28 \\
FIGO stage I & $106(71.6 \%)$ & $84(87.5 \%)$ & $26(50.0 \%)$ & $<0.05^{*}$ \\
Stage II & $7(4.7 \%)$ & $4(4.2 \%)$ & $3(5.8 \%)$ & \\
Stage III & $22(14.9 \%)$ & $6(6.3 \%)$ & $16(30.8 \%)$ & \\
Stage IV & $10(6.8 \%)$ & $2(2.1 \%)$ & $8(15.4 \%)$ & \\
Positive ascites cytology & $20 / 137(14.6 \%)$ & $8 / 91(8.8 \%)$ & $12 / 46(26.1 \%)$ & $<0.05^{*}$ \\
Lymph vascular space invasion & $30 / 109(27.5 \%)$ & $11 / 76(14.5 \%)$ & $19 / 36(52.8 \%)$ & $<0.05^{*}$ \\
Lymph node metastasis & $20 / 127(15.7 \%)$ & $7 / 84(8.3 \%)$ & $13 / 43(30.2 \%)$ & $<0.05^{*}$ \\
Recurrence & $28 / 148(18.96 \%)$ & $6 / 96(6.3 \%)$ & $22 / 52(42.3 \%)$ & $<0.05^{*}$ \\
Death & $21 / 148(14.2 \%)$ & $4 / 96(4.2 \%)$ & $17 / 52(32.7 \%)$ & $<0.05^{*}$ \\
Familial history of malignancy & $45 / 148(30.4 \%)$ & $30 / 96(31.3 \%)$ & $15 / 52(28.8 \%)$ & 0.85 \\
Complication with diabetes mellitus & $29 / 134(21.6 \%)$ & $20 / 90(22.2 \%)$ & $9 / 44(20.5 \%)$ & 0.58 \\
Complication with hypertension & $49 / 139(35.3 \%)$ & $34 / 91(37.4 \%)$ & $15 / 48(31.3 \%)$ & 1.00 \\
\hline
\end{tabular}

*Statistical significance $(p<0.05)$
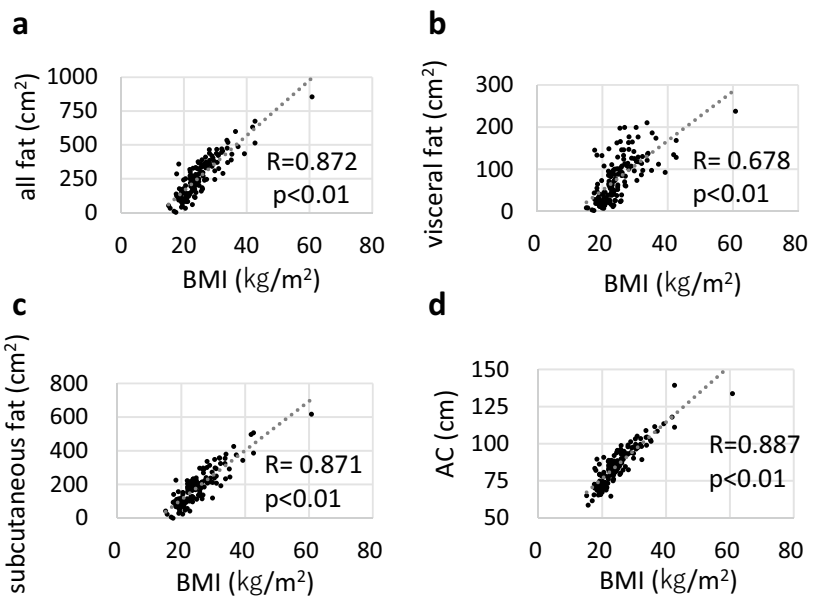

d

type 1 endometrial cancer, but not in type $2 p=0.0080$ and $p=0.8231$, respectively; Fig. 2e, f.

For OS, BMI and AC did not show statistically significant differences for type 1 and type 2 ( $p=0.1832, p=0.1644$ and $p=0.0738$ and $p=0.4824$, respectively; Fig. 3a-d). However, the $V / S$ ratio showed a statistically significant difference for type 1 but not type $2(p=0.0053$ and $p=0.4166$, respectively; Fig. 3e, f). We also analyzed obesity parameters using a COX regression univariate analysis of PFS and OS. For PFS, only the $V / S$ ratio was a significant prognostic factor ( $p=0.033$, Table 2$)$, whereas there were no significant prognostic factors for OS (Table 3).

\section{Discussion}

The results revealed that $V / S$ ratio $>0.5$ was a factor for poorer prognosis in cases of type 1 endometrial cancer. BMI is a major parameter of obesity, and although it is known to be risk factor for endometrial cancer [5-7], its relation with the prognosis has not been investigated sufficiently [11]. Obesity is divided into visceral-type obesity and subcutaneous-type obesity. The visceral type is more severe than the subcutaneous type because of its higher incidence of

Fig. 1 Scatter diagrams of obesity parameters. BMI shows positive correlations with all fat (a), visceral fat (b), subcutaneous fat (c), and AC (d), but not with $V / S$ ratio (e) 
Fig. 2 Kaplan-Meier curves of type 1 and type 2 endometrial cancers for PFS. Log-rank tests indicate that $\mathrm{BMI}$ and $\mathrm{AC}$ are not significant prognostic factors in both type 1 and 2 endometrial cancers (a-d), whereas the group with a high $V / S$ ratio shows poorer prognosis than the one with a low $V / S$ ratio in type 1 endometrial cancer (e), but not in type 2 endometrial cancer (f)
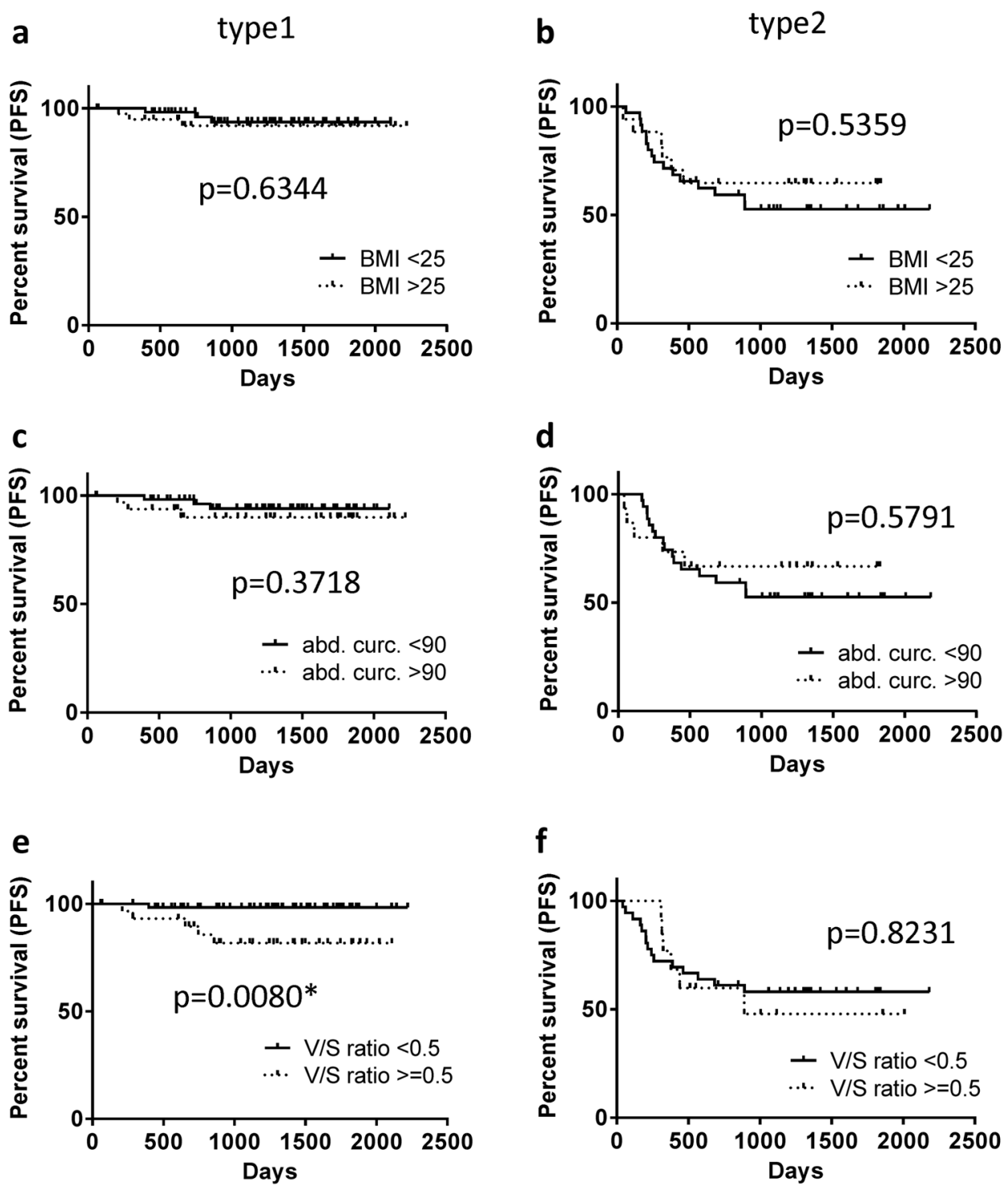

obesity-related diseases, such as diabetes mellitus, cerebrovascular disease, and ischemic heart disease [8].

To our knowledge, this is the first report to indicate that the $V / S$ ratio is related to OS and PFS of type 1 endometrial cancer. However, three previous studies have examined the $V / S$ ratio and prognostic factors in endometrial cancer. Ye et al. reported that in all types of endometrial cancer, $31.89 \%$ visceral adiposity (VAT\%) (equivalent to a $V / S$ ratio of 0.46 ) was a cutoff value for a significant difference in lymph node metastasis and extrauterine disease. They designated the median value of their study as the cutoff value. However, prognostic factors such as OS or PFS were not investigated [12].

Another study also used the median value of their study as a cutoff value. They found that VAT\% greater than $37 \%$ (equal to a $V / S$ ratio of 0.58 ) showed a negative prognostic impact on both PFS and OS in all types of endometrial cancer [13]. Cases with high visceral adiposity had higher mortality because of endometrial cancer [14]. However, the studies did not identify the difference between type 1 and type 2 endometrial cancers. Resent study identified that percentage of visceral out of total abdominal fat volume is associated with poor overall- and disease-specific survival in non-endometrioid endometrial cancer patients [15]. They did not use the $V / S$ ratio and not focused on type 1 endometrial cancer.

One report indicates that estradiol levels were not different regardless of menopausal status in type 1 and type 2 endometrial cancer [4]. However, our findings suggest that visceral fat is related to poor prognosis in only type 1 
Fig. 3 Kaplan-Meier curves of type 1 and type 2 endometrial cancers for OS. Log-rank tests indicate that BMI and $\mathrm{AC}$ are not significant prognostic factors in both type 1 and 2 endometrial cancers (a-d). Those with high $V / S$ ratio shows poorer prognosis than those with low $V / S$ ratio in type 1 endometrial cancer (e), but not in type 2 endometrial cancer (f) type1

a

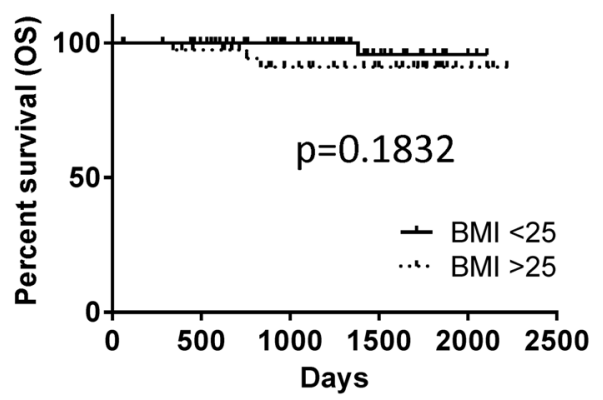

C

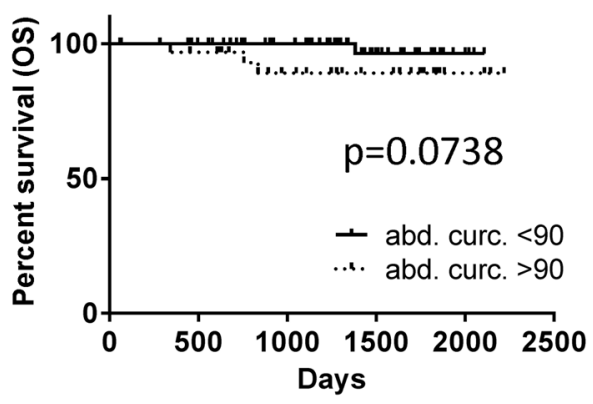

e

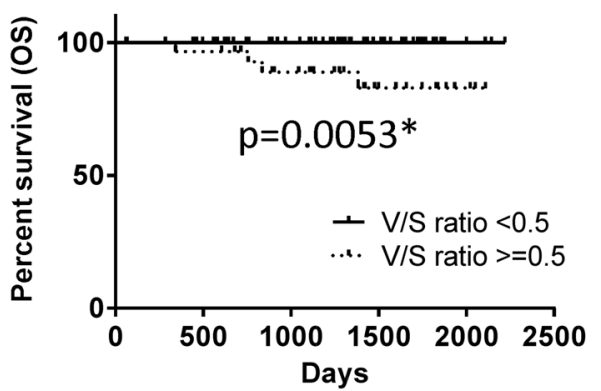

type2

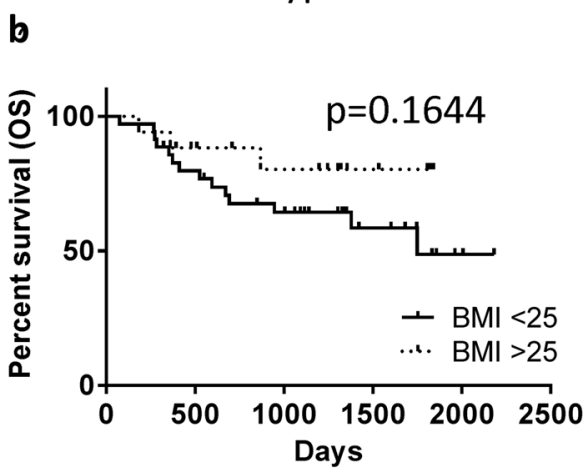

d
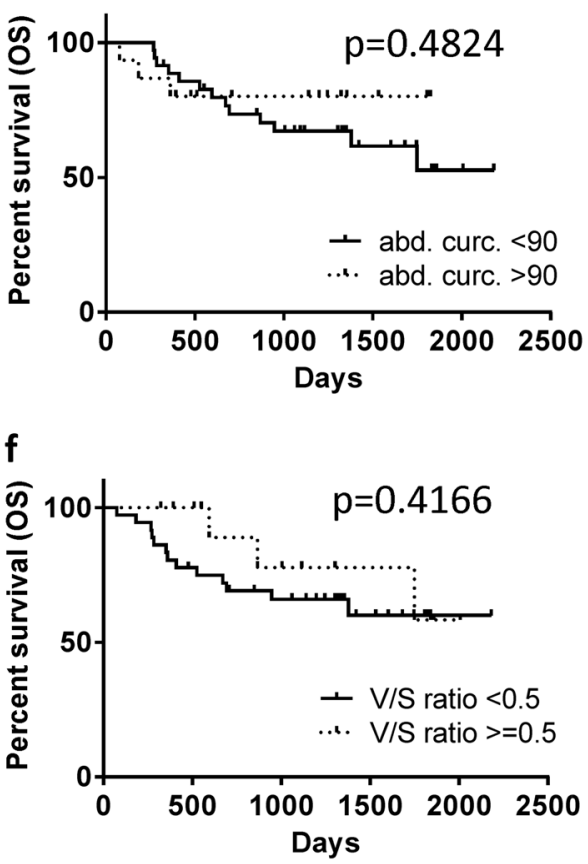

endometrial cancer, which is known to be estrogen dependent. Furthermore, a $V / S$ ratio of more than 0.4 is reported as a cutoff value for visceral-type obesity for cardiovascular disease [16]. In endometrial cancer, values of the $V / S$ ratio that are related to prognosis are higher than the $V / S$ ratio of 0.4 for detecting the risk of obesity-related diseases such as diabetes mellitus. This suggests that severe visceraltype obesity is a possible prognostic factor for endometrial cancer.

This study has some limitations. If the patients did not receive any therapies, they were excluded from this study, which means that it could be possible that the most advanced cases were not assessed. We did not perform lymphadenectomy in advanced stages. It should affect the prognosis. Furthermore, the Cox regression models did not show any statistical significance of the impact of poor clinical factors, including stage, ascites cytology, lymph vascular involvement, and lymph node metastasis. The possible reasons include a limited number of type 1 endometrial cancer patients, which show a favorable prognosis. We did not measured the serum levels of estradiol. Lastly, we did not validate the findings using another dataset.

In conclusion, a $V / S$ ratio $>0.5$ is a possible factor for poor prognosis in type 1 endometrial cancer. Nevertheless, further research is needed to investigate the preventive and therapeutic effects of the reduction of visceral fat on the prognosis of this type of cancer. 
Table 2 COX regression univariate analysis of progression-free survival in type 1 endometrial cancer patients

\begin{tabular}{|c|c|c|c|c|c|}
\hline & \multirow[t]{2}{*}{ PFS } & \multirow[t]{2}{*}{ Crude odds ratio } & \multicolumn{2}{|l|}{$95 \% \mathrm{CI}$} & \multirow[t]{2}{*}{$p$ value } \\
\hline & & & Lower & Upper & \\
\hline Age & $\begin{array}{l}\leqq 50, n=33 \\
>50, n=63\end{array}$ & 40.171 & 0.037 & $43,957.868$ & 0.301 \\
\hline BMI & $\begin{array}{l}<25, n=56 \\
\geqq 25, n=40\end{array}$ & 1.471 & 0.297 & 7.290 & 0.636 \\
\hline Abd. Circ & $\begin{array}{l}<90, n=58 \\
\geqq 90, n=33\end{array}$ & 1.929 & 0.421 & 0.421 & 0.421 \\
\hline$V / S$ ratio & $\begin{array}{l}<0.5, n=30 \\
\geqq 0.5, n=61\end{array}$ & 10.343 & 1.208 & 88.558 & $0.033^{*}$ \\
\hline FIGO stage & $\begin{array}{l}\text { I, } n=84 \\
\text { II-IV, } n=12\end{array}$ & 3.590 & 0.657 & 19.623 & 0.140 \\
\hline LVSI & $\begin{array}{l}\text { Absent, } n=65 \\
\text { Present, } n=11\end{array}$ & 6.746 & 0.422 & 107.959 & 0.177 \\
\hline Ascites & $\begin{array}{l}\text { Negative, } n=83 \\
\text { Positive, } n=8\end{array}$ & 0.042 & 0.000 & 4389.474 & 0.591 \\
\hline LNs metastasis & $\begin{array}{l}\text { Absent, } n=78 \\
\text { Present, } n=6\end{array}$ & 3.218 & 0.375 & 27.589 & 0.286 \\
\hline
\end{tabular}

*Statistical significance $(p<0.05)$

\begin{tabular}{|c|c|c|c|c|c|}
\hline & \multirow[t]{2}{*}{ PFS } & \multirow[t]{2}{*}{ Crude odds ratio } & \multicolumn{2}{|c|}{$95 \% \mathrm{CI}$} & \multirow[t]{2}{*}{$p$ value } \\
\hline & & & lower & upper & \\
\hline Age & $\begin{array}{l}\leqq 50, n=33 \\
>50, n=63\end{array}$ & 38.504 & 0.006 & $230,030.880$ & 0.411 \\
\hline BMI & $\begin{array}{l}<25, n=56 \\
\geqq 25, n=40\end{array}$ & 4.127 & 0.428 & 39.757 & 0.220 \\
\hline Abd. Circ & $\begin{array}{l}<90, n=58 \\
\geqq 90, n=33\end{array}$ & 5.687 & 0.591 & 54.678 & 0.132 \\
\hline$V / S$ ratio & $\begin{array}{l}<0.5, n=30 \\
\geqq 0.5, n=61\end{array}$ & 160.894 & 0.019 & $1,331,706.347$ & 0.270 \\
\hline FIGO stage & $\begin{array}{l}\text { I, } n=84 \\
\text { II-IV, } n=12\end{array}$ & 6.284 & 0.875 & 45.109 & 0.068 \\
\hline LVSI & $\begin{array}{l}\text { Absent, } n=65 \\
\text { Present, } n=11\end{array}$ & 6.573 & 0.411 & 105.146 & 0.183 \\
\hline Ascites & $\begin{array}{l}\text { Negative, } n=83 \\
\text { Positive, } n=8\end{array}$ & 0.041 & 0.000 & $25,209.750$ & 0.638 \\
\hline LNs metastasis & $\begin{array}{l}\text { Absent, } n=78 \\
\text { Present, } n=6\end{array}$ & 4.464 & 0.460 & 43.344 & 0.197 \\
\hline
\end{tabular}

*Statistical significance $(p<0.05)$
Table 3 COX regression univariate analysis of overall survival in type 1 endometrial cancer patients
Acknowledgements We thank Kazuya Muranaka who collected clinical data and the members of the division of radiology for their technical support.

\section{Declarations}

Conflict of interest No potential conflict of interest relevant to this article is reported.

Open Access This article is licensed under a Creative Commons Attribution 4.0 International License, which permits use, sharing, adaptation, distribution and reproduction in any medium or format, as long as you give appropriate credit to the original author(s) and the source, provide a link to the Creative Commons licence, and indicate if changes were made. The images or other third party material in this article are included in the article's Creative Commons licence, unless indicated otherwise in a credit line to the material. If material is not included in the article's Creative Commons licence and your intended use is not permitted by statutory regulation or exceeds the permitted use, you will need to obtain permission directly from the copyright holder. To view a copy of this licence, visit http://creativecommons. org/licenses/by/4.0/. 


\section{References}

1. Lortet-Tieulent J, Ferlay J, Bray F et al (2018) International Patterns and Trends in Endometrial Cancer Incidence, 1978-2013. J Natl Cancer Inst 110(4):354-361. https://doi.org/10.1093/jnci/ djx 214

2. Feingold KR, Anawalt B, Boyce A et al (2000) Endotext. PMID 25905390.

3. Setiawan VW, Yang HP, Pike MC et al (2013) Type I and II endometrial cancers: have they different risk factors? J Clin Oncol 31(20):2607-2618. https://doi.org/10.1200/JCO.2012.48.2596

4. Wan J, Gao Y, Zeng K et al (2016) The levels of the sex hormones are not different between type 1 and type 2 endometrial cancer. Sci Rep 6:39744. https://doi.org/10.1038/srep39744

5. Reeves GK, Pirie K, Beral V et al (2007) Cancer incidence and mortality in relation to body mass index in the Million Women Study: cohort study. BMJ 335(7630):1134. https://doi.org/10. 1136/bmj.39367.495995.AE

6. Wise MR, Jordan V, Lagas A et al (2016) Obesity and endometrial hyperplasia and cancer in premenopausal women: a systematic review. Am J Obstet Gynecol 214(6):689.e681-689.e617. https:// doi.org/10.1016/j.ajog.2016.01.175

7. Kyrgiou M, Kalliala I, Markozannes G et al (2017) Adiposity and cancer at major anatomical sites: umbrella review of the literature. BMJ 356:j477. https://doi.org/10.1136/bmj.j477

8. Prabhakaran D, Anand S, Gaziano TA et al (2017) Cardiovascular, respiratory, and related disorders. PMID 30212077

9. Smrz SA, Calo C, Fisher JL et al (2020) An ecological evaluation of the increasing incidence of endometrial cancer and the obesity epidemic. Am J Obstet Gynecol. https://doi.org/10.1016/j.ajog. 2020.10.042
10. Ciortea R, Mihu D, Mihu CM (2014) Association between visceral fat, IL-8 and endometrial cancer. Anticancer Res 34(1):379-383

11. Arem H, Irwin ML (2013) Obesity and endometrial cancer survival: a systematic review. Int J Obes (Lond) 37(5):634-639. https://doi.org/10.1038/ijo.2012.94

12. Ye S, Wen H, Jiang Z et al (2016) The effect of visceral obesity on clinicopathological features in patients with endometrial cancer: a retrospective analysis of 200 Chinese patients. BMC Cancer 16:209. https://doi.org/10.1186/s12885-016-2230-4

13. Mauland KK, Eng $\varnothing$, Ytre-Hauge $S$ et al (2017) High visceral fat percentage is associated with poor outcome in endometrial cancer. Oncotarget 8(62):105184-105195. https://doi.org/10.18632/oncot arget. 21917

14. Celik E, Kizildag Yirgin I, Goksever Celik H et al (2020) Does visceral adiposity have an effect on the survival outcomes of the patients with endometrial cancer? J Obstet Gynaecol Res. https:// doi.org/10.1111/jog. 14560

15. Donkers H, Fasmer KE, Mcgrane J et al (2021) Obesity and visceral fat: survival impact in high-grade endometrial cancer. Eur J Obstet Gynecol Reprod Biol 256:425-432. https://doi.org/10. 1016/j.ejogrb.2020.11.050

16. Matsuzawa Y, Nakamura T, Shimomura I et al (1995) Visceral fat accumulation and cardiovascular disease. Obes Res 3(Suppl 5):645S-647S. https://doi.org/10.1002/j.1550-8528.1995.tb004 81.x

Publisher's Note Springer Nature remains neutral with regard to jurisdictional claims in published maps and institutional affiliations. 